\title{
Application and Analysis of GOOSE Protocol in Industrial Process Controller
}

\author{
Honghai Niu, Jun Chen and Liu Liu \\ NR Electric Co., Ltd.Nanjing, 211102, China
}

\begin{abstract}
In application of industrial process control, more and more equipment \& devices are integrated, and then data transmission encounters a serious bottleneck. Reliable \& rapid data transmission between industrial process controllers \& IO units becomes very urgent. Many schemes have been presented to solve this problem, but each of them has its own shortcomings. In this paper, a new scheme based on GOOSE (Generic Object Oriented Substation Event) is designed to promote data transfer rate \& system reliability. Moreover, mass data transmission can also be completed, and messages with the length up to 1518 bytes are acceptable. This scheme has been applied in many projects and proven effective.
\end{abstract}

Keywords-GOOSE; Basic Encoding Rules; ASN.1; Industrial Process Controller; Real-time and Reliability

\section{INTRODUCTION}

GOOSE (Generic Object Oriented Substation Event) is widely applied in digital substations which adopt IEC61850 protocol $^{[1]}$. Distributed bus relay, ATS (Auto-Transfer Switch) \& load shedding have been implemented in many projects ${ }^{[2]}$.

In paper [3], a CNC (Computer Numerical Controller) is designed to realize a scheme of " 1 master unit \& multiple slave units". In this scheme, the master unit request data from the slave units, but sudden data change can not be sent to the master unit until the master unit requests data from the slave units.

In paper [4], a new controller based on ControlNet is provided, but the length of available ControlNet message can not exceed 510 bytes $^{[5]}$.

In paper [6], a new controller based on DeviceNet is provided, but mass data transmission can not be treated because short frame is adopted in DeviceNet.

In this paper, a new controller based on GOOSE protocol is designed, sudden data change can be transmitted, and max length of available message is 1518 bytes. Therefore, all the shortcomings in paper [3] [6] will be solved perfectly in this paper.

\section{Methodology}

In this scheme, 2 industrial process controllers (abbreviated as controller hereinafter) are designed, one works as the master controller, while the another works as the slave controller. Meanwhile, dual networks are adopted. Master/Slave controller communicates with the IO units via dual networks (net A \& net B).
Only the master controller can transmit control command to IO unit via net A \& net B.

The slave controller only exchanges heartbeat messages with the IO unit, so as to judge communication states between the controller \& IO unit.

If the slave controller is switched to the master controller, the former "master controller" will not transmit control command, i.e.: only the new master controller will send transmit control command to the IO unit.

The master controller transmits heartbeat request messages to the IO units via net A \& net B. The IO units will differentiate whether it is a net $\mathrm{A}$ or net $\mathrm{B}$ heartbeat request message, and then reply a heartbeat response to the master controller.

The master controller transmit GOOSE control messages to IO units via net $A$ \& net $B$, and then the IO units will judge whether to execute this message or not.

System structure is shown in FIGURE I.

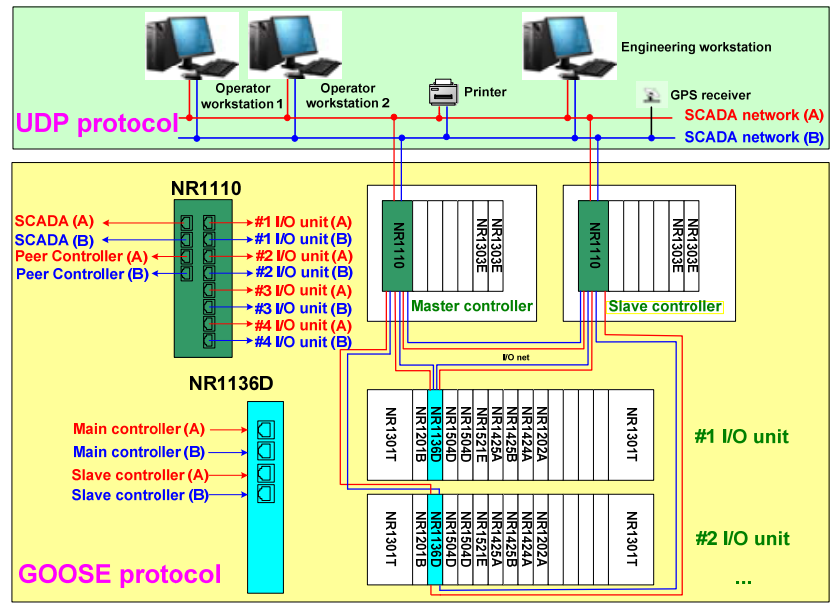

FIGURE I. SYSTEM STRUCTURE

\section{MeChanism Of GoOse Messages}

\section{A. Introduction of GOOSE Messages}

By implementing the GOOSE service model defined in IEC61850-7-2, input/output data can be transmitted quickly. To ensure real-time performance \& reliability ${ }^{[7-9]}$, a special re-transmission mechanism is provided: GOOSE protocol stack as shown in FIGURE II.

Main features of GOOSE protocol stack is listed as below: 
1) Only 4 layers of OSI mode are adopted: Application layer, Presentation layer, Data link layer \& Physical layer. Therefore, reliability of data transmission can be promoted, and transmission time delay can be minimized.

2) IEEE802.1Q protocol is adopted in Data link layer, GOOSE protocols can be transmitted in priority. Therefore, GOOSE network security is ensured.

3) GOOSE communication protocol is based on peer-to-peer (P2P) mechanism, maintenance cost is reduced effectively.

4) For GOOSE communication protocol in application level, besides data validity check, re-transmission mechanism in case of GOOSE message loss is provided to ensure reliable transmission.

5) Fiber optic cable \& Ethernet twisted-pair cable are adopted in GOOSE transmission, the data transfer rate can reach $100 \mathrm{M} / 1000 \mathrm{Mbps}$, which can fully meet GOOSE transmission requirements in industrial process control.

\begin{tabular}{|c|c|c|}
\hline & \multicolumn{2}{|c|}{ GOOSE } \\
\hline Application layer & \multicolumn{2}{|c|}{ IEC61850-8-1 } \\
\hline Presentation layer & \multicolumn{2}{|c|}{ ASN.1/BER } \\
\hline \multicolumn{3}{|l|}{ Session layer } \\
\hline \multicolumn{3}{|l|}{ Transport layer } \\
\hline \multicolumn{3}{|l|}{ Network layer } \\
\hline Data link layer & \multicolumn{2}{|c|}{ Ethernet/IEEE802.1Q } \\
\hline Physical layer & Optic fiber & Twisted-pair \\
\hline
\end{tabular}

FIGURE II. GOOSE PROTOCOL STACK

\section{B. ASN.1 Code}

In presentation layer, BER (Basic Encoding Rules) of ASN.1 is applied, data will be transmitted/received in data link layer directly (instead of Transport layer \& Network layer). In this way, protocol treatment time is $\operatorname{minimized}^{[2]}$. Format of $\mathrm{T}-\mathrm{L}-\mathrm{V}$ is applied in BER:
1) $\mathrm{T}: \quad$ Type;
2) L: Length;
3) V: Value.

Note: "V" can also be the combination of "T-L-V" as shown in FIGURE III.

\begin{tabular}{|c|c|c|c|c|c|c|c|c|}
\hline \multicolumn{2}{|c|}{ T } & L & \multicolumn{2}{|c|}{ V } \\
\hline \multicolumn{2}{|c|}{ Type (HEX) } & Length (HEX) & \multicolumn{2}{c|}{ Value (HEX) } \\
\hline
\end{tabular}

FIGURE III. FORMAT OF T-L-V
In BER, "L" (Length) will be influenced by "V"(Value). Every time in transmitting/receiving the same data, change of "V"(Value) will lead to change of "L", so "L" can not be pre-configured.

Therefore, in BER, "V" will be determined first, and then "L” \& "T" will be added to the code sequentially.

\section{SOFTWARE PRINCIPLE}

\section{A. Mechanism of GOOSE Message Transmission}

In this paper, GOOSE communication is based on board, i.e.: data of the whole board is regarded as a communication unit during GOOSE data transmission.

Different from Client/Server mode, Subscriber/Publisher mode is adopted, so as to enhance data transfer rate and minimize network resource consumption ${ }^{[11]}$.

In case of heavy network load, to avoid GOOSE message loss,GOOSE re-transmission mechanism is adopted to ensure reliability of data transfer.

During GOOSE data transmission, GOOSE message will be transmitted 5 times (Repeated 4 times: 1ms, 2ms, $4 \mathrm{~ms} \& 8 \mathrm{~ms}$ after first transmission):

If the data remain unchanged, it will be re-transmitted every 5 seconds;

If the data change, it will be re-transmitted 5 times immediately (Repeated 4 times: 1ms, 2ms, $4 \mathrm{~ms} \& 8 \mathrm{~ms}$ after first transmission).

\section{In GOOSE transmission:}

[StateNo.] is used to indicate message content No., i.e.: if the message content is the same to last message, [StateNo.] will remain unchanged. If the message content is different from last message, [StateNo.] will increase 1 (i.e.: “[StateNo.]++" in FIGURE IV

[SequenceNo.] is used to indicate message re-transmission No., i.e.: if a GOOSE message is transmitted for the first time, [SequenceNo.] $=0$; if a GOOSE message has been re-transmitted for 5 times, [SequenceNo.] $=4$.

Dual networks are adopted between industrial process controller \& IO units. It is necessary to identify whether received data is new data, or just the same data received via the other network, so as to decide to receive or discard.

To solve this problem, the field [RedundancyNo.] is used to represent GOOSE transmission message No.

Flow chart of dual net GOOSE transmission is shown in FIGURE IV as below. 


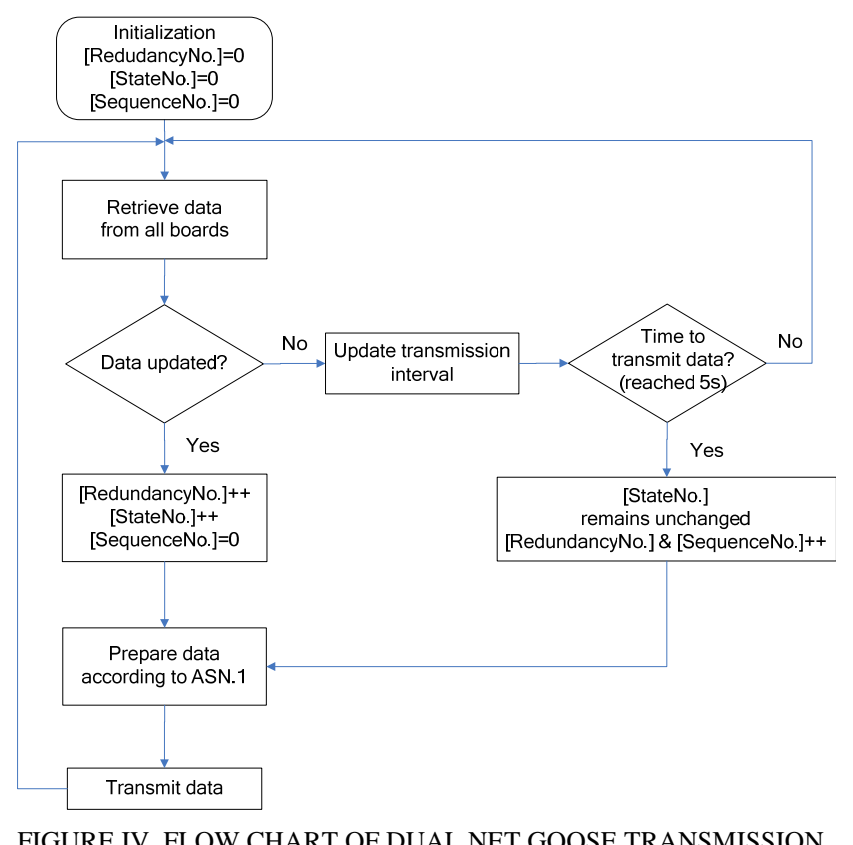

B. Mechanism of GOOSE Message Reception

Flow chart of dual net GOOSE reception is shown in FIGURE $V$ as below.

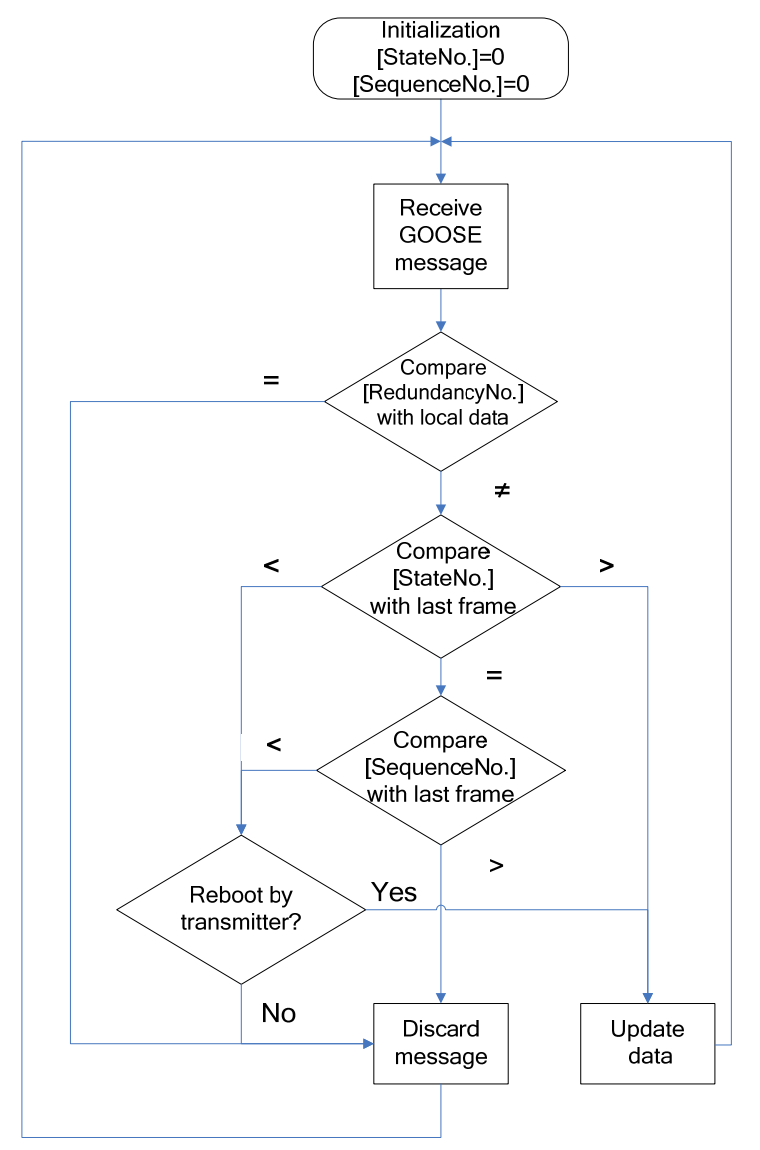

FIGURE V. FLOW CHART OF DUAL NET GOOSE RECEPTION

\section{EXPERIMENTAL RESULTS AND ANALYSIS}

A. Analysis of Real-time Performance

Message transfer time is defined in IEC61850 protocol, and it is analyzed in the following figure.

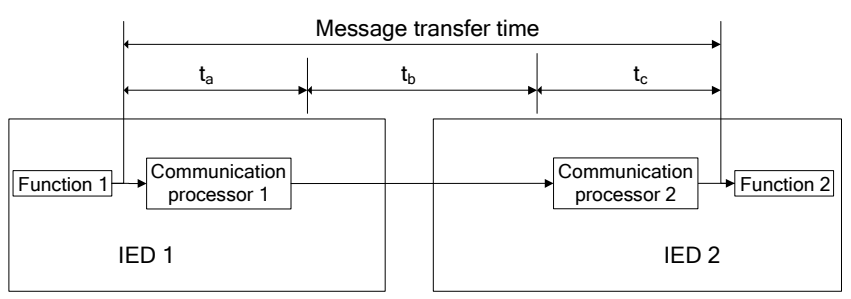

FIGURE VI. MESSAGE TRANSFER TIME

As shown in FIGURE VI:

1) ta: Message transmission time;

2) tb: Network time delay;

3) tc: Message reception time ${ }^{[12][13]}$.

ta \& tc depends on performance of communication processors, they will not be introduced in this paper. Only tb will be analyzed here.

tb consists of the following 4 parts:

1) t_sf:

Time delay of storage \& forwarding.

t_sf can be concluded by "Message length/data transfer rate", e.g.: for $100 \mathrm{Mbps}$ Ethernet switch, $122 \mu \mathrm{s}$ is required to store \& forward a GOOSE message with 1518 bytes.

2) t_latency:

Time delay of Ethernet switch latency.

Time delay of Ethernet switch latency is determined by processing capability of corresponding chip. Generally speaking, $\mathrm{t}$ _latency of industrial $100 \mathrm{Mbps}$ Ethernet switch is less than $10 \mu \mathrm{s}$.

3) t_fiber:

Time delay of optical fiber transmission.

t_fiber can be concluded by "Optical fiber length/Light velocity in the cable”, e.g.: for $1 \mathrm{~km}$ optic fiber, t_fiber is about $5 \mu \mathrm{s}$.

4) t_queue:

Time delay of Ethernet switch queue.

FIFO (First In, First Out) mechanism is adopted in processing Ethernet switch message queue.

Suppose number of Ethernet ports is K.

In the worst condition, we must wait data transmission of all the other Ethernet ports, i.e.:

t_queue $=(\mathrm{K}-1) * \mathrm{t} \_s f$ 
In the best condition, we do not need to wait any Ethernet port, this message can be transmitted immediately:

\section{$\mathrm{t} \_$queue $=0$}

Average of t_queue is “(K-1)*t_sf/2”.

Suppose:

It is a $100 \mathrm{Mbps}$ Ethernet switch with 24 ports

(i.e.: $\mathrm{K}=24$ ),

GOOSE message length=1518bytes,

Optical fiber length=1km;

So:

$$
\begin{aligned}
& \text { tb }=\text { t_sf }+ \text { t_latency }+ \text { t_fiber }+ \text { t_queue } \\
& =122+10+5+(24-1) * 122 / 2 \\
& =1540 \mu \mathrm{s}
\end{aligned}
$$

In the experiment in paper [14]:

Maximum GOOSE message transfer time is $1500 \mu \mathrm{s}$,

Minimum GOOSE message transfer time is $900 \mu \mathrm{s}$,

Average GOOSE message transfer time is $1170 \mu \mathrm{s}$.

The result is consistent with theoretical analysis in this paper.

\section{B. Analysis of Reliability}

Dual networks are adopted between industrial process controller \& IO units, i.e.: even if one network is disconnected, communication between controllers \& IO units can still work normally.

Moreover, dual GOOSE networks operate together (not work in host/standby mode), so there is no time delay by network switchover.

Based on above analysis, reliability of network transmission is achieved.

\section{CONCLUSION}

By application of this scheme, rapid \& reliable data transmission can be realized by adopting industrial process controller based on GOOSE protocol. Moreover, stability \& reliability of industrial process control system can be promoted greatly.

GOOSE protocol has been widely applied in power system automation, but never adopted in industrial process control.

In application of industrial process control, PCS-9150 process controller manufactured by NR is the first product which implements GOOSE protocol.

This scheme has proven effective by applications of PCS-9150 in various projects.

\section{REFERENCES}

[1] DL/T 860.72-2004 Part7-2:Basic communication structure for substation and feeder equipment Abstract communication service interface(ACSI).
[2] SONG Li-jun, WANG Ruo-xing, and DI Jun-feng, "Analysis and implementation of GOOSE mechanism and its application in digital substation,” Power System Protection and Control, vol. 37, no. 14, pp. 31-34, 2009.

[3] WANG Zhicheng, YU Dong, and ZHANG Xiaohui, "Study on Reliable Communication Mechanism of Field-bus for Numerical Control System," Journal of Mechanical Engineering, vol. 47, no. 3, pp. 152-158, 2011.

[4] Zhu Jun, Wang Jinkuan, and An Cuijuan, "Network analyze of Enther Net IP based on redundancy ControlNet," Chinese Journal of Scientific Instrument, vol. 27, no. 6, pp.698-700, 2006.

[5] FANG Xiao-ke, WANG Jian-hui, andQIAN Xiao-long, "Analysis of media access control for ControlNet fiedbus," Control and Desision, vol. 19, no. 9, pp. 1054-1056, 2004.

[6] FANG Xiao-ke, XU Lin, and WANG Jian-hui, “Analysis of DeviceNet Character and Design of Its Node,” Chinese Journal of Scientific Instrument, vol. 26, no. 11, pp.1174-1177, 2005.

[7] ZHU Bin-quan, and WANG Song, “Application of IEC 61850 GOOSE Technology on Protective Relaying," Automation of Electric Power Systems, vol. 33, no. 8, pp. 104-107, 2009.

[8] YIN zhi-liang, LIU Wan-shun and YANG Qi-xun, "Generic Substation Event Model Based on IEC61850,” Automation of Electric Power Systems, vol. 29, no. 19, pp. 45-50, 2005.

[9] FAN Jianzhong and MA Qianli, "GOOSE and its Application," Automation of Electric Power Systems, vol. 31, no. 19, pp. 85-90, 2007.

[10] Zheng Wei-guang, Wan Yutao andWang Wei, "Implementation and Application of BER-Based Asn.1 Syntax Complier and Encoder Decoder," Computer Application and Software, vol. 28, no. 2, pp. 195-198, 2011.

[11] XU Chengbin and SUN Yimin, "A Communication Solution of Process Layer GOOSE in Digitized Substation,” Automation of Electric Power Systems, vol. 31, no. 19, pp. 91-94, 2007.

[12] FU Xuhua, HUANG Xiaoming and WANG Song, "Engineering application of GOOSE technology in digital substation,” Electric Power Automation Equipment, vol. 31, no. 11, pp. 112-115, 2011.

[13] WANG Song and LU Chengyu, “A GOOSE Network Scheme for Relay Protection in Digitized Substations," Automation of Electric Power Systems, vol. 33, no. 3, pp. 51-54, 2009.

[14] ZHENG Xin-cai, ZHOU Xin, and WANG Su-hua, "Research on test technology of GOOSE network in digital substation", Power System Protection and Control, vol. 37, no. 24, pp. 85-89, 2009. 